\title{
Restricted Isometry Property of Principal Component Pursuit with Reduced Linear Measurements
}

\author{
Qingshan You, ${ }^{1,2}$ Qun Wan, ${ }^{2}$ and Haiwen $\mathrm{Xu}^{1}$ \\ ${ }^{1}$ School of Computer Science, Civil Aviation Flight University of China, GuangHan, Sichuan 618307, China \\ ${ }^{2}$ School of Electronic Engineering, University of Electronic Science and Technology of China, Chengdu, Sichuan 611731, China
}

Correspondence should be addressed to Qun Wan; wanqun@uestc.edu.cn

Received 25 September 2012; Revised 2 April 2013; Accepted 30 April 2013

Academic Editor: Xiaojun Wang

Copyright (C) 2013 Qingshan You et al. This is an open access article distributed under the Creative Commons Attribution License, which permits unrestricted use, distribution, and reproduction in any medium, provided the original work is properly cited.

The principal component prsuit with reduced linear measurements (PCP_RLM) has gained great attention in applications, such as machine learning, video, and aligning multiple images. The recent research shows that strongly convex optimization for compressive principal component pursuit can guarantee the exact low-rank matrix recovery and sparse matrix recovery as well. In this paper, we prove that the operator of PCP_RLM satisfies restricted isometry property (RIP) with high probability. In addition, we derive the bound of parameters depending only on observed quantities based on RIP property, which will guide us how to choose suitable parameters in strongly convex programming.

\section{Introduction}

Matrix completion is a signal processing technique, which recovers an unknown low-rank or approximately low-rank matrix from a sampling of its entries. Recently, this technique has been applied in many fields, such as medical [1], imaging [2], seismology [3], and computer vision [4-6]. The dimension of data produced in practice is always vastly high, therefore, there exists a pressing challenge to develop efficient and effective tools to process, analyze, and extract useful information from a small set of linear measurements of the high-dimensional data. Many scholars had studied in detail in the recent papers [7-13]. Candès et al. address the problem of recovery of the unknown low-rank matrix and sparse matrix from the sampling of its complete entries [7-9] for large scale. As the extensions of previous works, Ganesh et al. address the problem of decomposing a superposition of a low-rank matrix and a sparse matrix when a relatively few linear measurements are available $[10,11]$.

These fundamental results have a great impact in engineering and applied mathematics. However, these results are limited into convex optimization; that is, nuclear norm based convex optimization leads to the exact low-rank matrix recovery under suitable conditions. It is well known that strongly convex optimization has many intrinsic advantages, such as the uniqueness of optimal solution. Especially, the strongly convex optimization was widely used in designing efficient and effective algorithm in the literature of compressive sensing and low-rank matrix recovery. Zhang et al. extend them; they proved that strongly convex optimizations can guarantee the exact low-rank matrix recovery as well in the paper [12]. The right bound of $\tau$ of strongly convex programming for robust principal component analysis was provided in paper [14].

Pertaining to the strongly convex optimization for compressive principal component pursuit, we had studied it in detail in our former work in [15], in which we prove that under suitable conditions, the corresponding strongly convex optimization also recovers the low-rank matrix and sparse matrix exactly. However, the bound of parameter strongly depends on the Frobenius norm of the data, which is not known in practice, because the data we can use is $\mathscr{P}_{\mathrm{Q}} M$ only. In this paper, we will give an easy band of $\tau$ depending on $\mathscr{P}_{\mathrm{Q}} M$ only.

1.1. Contents and Notations. For convenience, we will give here a brief summary of the notations that will be used throughout the paper. We denoted the operator norm of matrix by $\|X\|$, the Frobenius norm by $\|X\|_{F}$, the nuclear 
norm by $\|X\|_{*}$ and the $l_{\infty}$ norm by $\|X\|_{\infty}:=\max _{i j}\left|x_{i j}\right|$. We will bound the Euclidean inner product between two matrices which is defined by the formula $\langle X, Y\rangle=\operatorname{trace}\left(X^{*} Y\right)$. The Cauchy-Schwarz inequality gives $\langle X, Y\rangle \leq\|X\|_{F}\|Y\|_{F}$, especially $\|X\|_{*} \leq \sqrt{r}\|X\|_{F}$ and $\sum_{i j}\left|x_{i j}\right| \leq \sqrt{|X|_{0}}\|X\|_{F}$. We will also bound linear transformations which act on the space of matrices, and we denote these operators by $\mathscr{P}_{T} X$. The norm of the operator is denoted by $\|\mathscr{P}\|$; that is, $\|\mathscr{P}\|=$ $\sup _{\left\{\|X\|_{F}=1\right\}}\|\mathscr{P} X\|_{F}$. We denote by $\Omega$ the support of $S_{0}$. By a slight abuse of notation, we also represent by $\Omega$ the subspace of matrices whose support is contained in the support of $S_{0}$. $\operatorname{Supp}\left(S_{0}\right) \sim \operatorname{Ber}(\rho)$ means each entry of the matrix belongs to the support of the sparse matrix $S_{0}$ independently with probability $\rho$.

1.2. Basic Problem and Main Result. In this paper, we only address the strongly convex programming of principal component pursuit with reduced linear measurements, which is considered in paper [10]. This problem has the following formula:

$$
\begin{aligned}
& \operatorname{minimize}\|L\|_{*}+\lambda\|S\|_{1}+\frac{1}{2 \tau}\|L\|_{F}^{2}+\frac{1}{2 \tau}\|S\|_{F}^{2} \\
& \text { subject to } \mathscr{P}_{\mathrm{Q}} M=\mathscr{P}_{\mathrm{Q}}(L+S),
\end{aligned}
$$

where $\tau>0$ is some positive penalty parameter and $P_{\mathrm{Q}}$ is the orthogonal projection onto the linear subspace $Q$, and $Q$ is distributed according to the Haar measure on the Grassmannian $\mathbb{G}\left(R^{m \times n} ; q\right)$. Suppose that $G_{1}, G_{2}, \ldots, G_{p}$ are standard orthogonal basis of $Q^{\perp}$; that is, $G_{1}, G_{2}, \ldots, G_{p}$ are identical in distribution to $G$, where $G=H /\|H\|_{F}$ and $H=$ $M / \sqrt{m n}$, whose entries are i.i.d, according to the standard normal distribution. More detail was interpreted in the paper [10].

When $\tau=\infty$ in the problem (1), we have existence and uniqueness theorems as follows.

Theorem 1 (see [10]). Fix any $C_{p}>0$, and let $Q^{\perp}$ be a $p$ dimensional random subspace of $\mathbb{R}^{m \times n} ; L_{0}$ obeys incoherence condition with parameter $\mu$, and $\operatorname{supp}\left(S_{0}\right) \sim \operatorname{Ber}(\rho)$. Then, with high probability, the solution of problem (1) with $\lambda=1 / \sqrt{n}$ is exact; that is, $\widehat{L}=L_{0}$ and $\widehat{S}=S_{0}$, provided that

$$
\operatorname{Rank}\left(L_{0}\right)<C_{r} n \mu^{-1}(\log m)^{-2}, \quad p<C_{p} n, \quad \rho<\rho_{0},
$$

where, $C_{r}, C_{p}$ and $\rho_{0} \in(0,1)$ are positive numerical constants.

Remark 2. The value of $\rho_{0}$ is a fixed positive numerical constant and should obey some suitable conditions; one of those is less than $1 / 5$. the detail derivation has been wellstudied (see, e.g., $[8,10]$ and the many references therein). With high probability means with probability at least $1-m^{-c}$, with $c>0$ numerical constant.

When $\tau \neq \infty$ in the problem (1), we have existence and uniqueness theorems as follow.
Lemma 3 (see $[14,15])$. Assuming

$$
\tau \geq \frac{8 \sqrt{15}\|M\|_{F}}{3 \lambda}
$$

under the other assumptions of Theorem $1,\left(L_{0}, S_{0}\right)$ is the unique solution to the strongly convex programming (1) with high probability.

Pertaining to the projection operator $\mathscr{P}_{\mathrm{Q}}$, we obtain the following restricted isometry property of operator $\mathscr{P}_{\mathrm{Q}}$ restricted in a superposition of low-rank matrices and sparse matrices.

Theorem 4. Suppose that $L$ is low-rank matrix with rank $r$, $S$ is sparse matrix with $\operatorname{Supp}\left(S_{0}\right) \sim \operatorname{Ber}(\rho), \rho \leq \delta^{2} /(64 \times$ $25 p \log (m n p))$, and $r \leq\left(m n \delta^{2} /(12 \times 25 p)\right)-(5 / 3) \log (n)$, and assume that the conditions of Theorem 1 hold. Then, with high probability, one has

$$
(1-\delta)\|M\|_{F} \leq\left\|\mathscr{P}_{\mathrm{Q}} M\right\|_{F} \leq(1+\delta)\|M\|_{F} .
$$

According to Lemma 3 and Theorem 4, we can easy obtain the following theorem.

Theorem 5. Supposing that $L$ is low-rank matrix with rank $r$, $S$ is sparse matrix with $\operatorname{Supp}(S) \sim \operatorname{Ber}(\rho)$. If

$$
\tau \geq \frac{8 \sqrt{15}\left\|\mathscr{P}_{\mathrm{Q}} M\right\|_{F}}{3(1-\delta) \lambda}
$$

under the other assumptions of Theorem $4,\left(L_{0}, S_{0}\right)$ is the unique solution to the strongly convex programming (1) with high probability.

It is obvious that the band of $\tau$ depends on $\mathscr{P}_{\mathrm{Q}} M$ only, which is obtained very easily in practice.

1.3. Contributions and Organization. In this paper, we first prove that although the operator $\mathscr{P}_{\mathrm{Q}}$ does not satisfy the restricted isometry property (RIP) in entire space, it obeys restricted isometry property (RIP) with high probability if the rank of low-rank matrix is not large and the sparse matrix is enough sparse. Second, we will use the restricted isometry property to improve the band of parameter which just depends on $\mathscr{P}_{\mathrm{Q}} M$.

The main center of the paper provides the proof of Theorem 4. The rest of paper is organized as follows. In Section 2, we will provide some important lemmas which are important composition of our main result and then will give the proof of Theorem 4. Conclusion and further works are discussed in Section 3.

\section{The Proof of Theorem 4}

In this section, we will provide the proof of Theorem 4 . We first bound the behaviors of the operator $\mathscr{P}_{\mathrm{Q}^{+}}$implying on the space of low-rank matrices and the space of sparse matrices, respectively. Then, the relation of $\|M\|_{F}$ and $\|L\|_{F}+\|S\|_{F}$, we can obtain Theorem 4. 
2.1. Bounding the Behavior of $\left\|\mathscr{P}_{\mathrm{Q}^{\perp}}(S)\right\|_{F}$. We will provide some important lemmas, which are used in the main theorem.

Lemma 6 (see [10, Lemma 6]). Let $Q^{\perp}$ be a linear subspace distributed according to the random subspace model described earlier. Then, for any $(i, j) \in[m] \times[n]$, with high probability

$$
\left\|\mathscr{P}_{\mathrm{Q}^{+}} e_{i} e_{j}^{T}\right\|_{F} \leq 4 \sqrt{\frac{p \log (m n p)}{m n}} .
$$

Bernstein's Inequality [16, 17]. Assume that $X_{1}, X_{2}, \ldots, X_{n}$ are independent random variables with $\mathbb{E}\left[X_{i}\right]=0$, for all $i$. Furthermore, suppose that $\left|X_{i}\right| \leq M$. Then,

$$
\mathbb{P}\left\{\left|\sum_{i} X_{i}\right|>t\right\} \leq 2 \exp \left(-\frac{t^{2}}{2 \sum_{i} \operatorname{Var}\left(X_{i}\right)+2 M t / 3}\right) .
$$

Lemma 7. If $\rho \geq \log n / m n$, then with high probability

$$
\|S\|_{0} \leq 2 \rho m n .
$$

Proof. Let $\omega_{i j}$ be the indicator variables with $\omega_{i j}=1$ if $(i j) \in \Omega$ and $\omega_{i j}=0$ for otherwise. For $S \in \Omega$, we have

$$
\|S\|_{0} \leq \sum_{i j} \omega_{i j}
$$

Let $Y_{i j}:=\omega_{i j}-\rho$ and $Z:=\sum_{i j} Y_{i j}$. For $\mathbb{E}\left[Y_{i j}\right]=\mathbb{E}\left[\omega_{i j}\right]-$ $\rho=0$ and $\left|Y_{i j}\right| \leq 1$, it is obvious that $Y_{i j}$ satisfies Bernstein's inequality. Note that

$$
\sum_{i j} \operatorname{Var}\left(Y_{i j}\right)=\rho(1-\rho) m n \leq \rho m n .
$$

According to Bernstein's inequality, letting $t=\rho m n$, we have

$$
\mathbb{P}\left\{\left|\sum_{i j} \omega_{i j}-\rho m n\right|>\rho m n\right\} \leq 2 \exp \left(-\frac{3 \rho m n}{8}\right) .
$$

Combining with $\rho \geq \log n / m n$, we have

$$
\mathbb{P}\left\{\left|\sum_{i j} \omega_{i j}-\rho m n\right|>\rho m n\right\} \leq n^{-3 / 8} .
$$

In other words, $\|S\|_{0} \leq 2 \rho m n$ with high probability. Note that $\lim _{n \rightarrow \infty} \log n / m n=0$. That means $\rho$ can obtain very small value when $n$ is very large.

Theorem 8(a). Suppose that $S$ is sparse matrix with $\operatorname{Supp}(S) \sim \operatorname{Ber}(\rho)$ and $Q^{\perp}$ is a p-dimensional random subspace of $\mathbb{R}^{m \times n}$. If $\rho \leq \delta^{2} / 64 p \log (m n p)$, with high probability, one has

$$
\left\|\mathscr{P}_{\mathrm{Q}^{\perp}} S\right\|_{F} \leq \delta\|S\|_{F} .
$$

Proof. According to triangle inequality, we have

$$
\begin{aligned}
\left\|\mathscr{P}_{\mathrm{Q}^{\perp}} S\right\|_{F} & =\left\|\mathscr{P}_{\mathrm{Q}^{\perp}}\left(\sum_{i j} S_{i j} e_{i} e_{j}^{T}\right)\right\|_{F} \\
& \leq \sum_{i j}\left|S_{i j}\right|\left\|\mathscr{P}_{\mathrm{Q}^{\perp}} e_{i} e_{j}^{T}\right\|_{F} \\
& \leq 4 \sqrt{\frac{p \log (m n p)}{m n}} \sum_{i j}\left|S_{i j}\right| \\
& \leq 2 \sqrt{\rho m n}\|S\|_{F} 4 \sqrt{\frac{p \log (m n p)}{m n}} \\
& \leq \sqrt{64 p \rho \log (m n p)}\|S\|_{F} .
\end{aligned}
$$

In the third inequality, we have used Lemma 6 . In the foarth inequality, we have used Cauchy-Schwarz inequality and Lemma 7. Combining with $\rho \leq \delta^{2} / 64 p \log (m n p)$, we can obtain

$$
\left\|\mathscr{P}_{\mathrm{Q}^{\perp}} S\right\|_{F} \leq \delta\|S\|_{F} .
$$

Theorem 8(b). Suppose that $S$ is sparse matrix with $\operatorname{Supp}(S) \sim \operatorname{Ber}(\rho)$, and $Q^{\perp}$ is a p-dimensional random subspace of $\mathbb{R}^{m \times n}$. If $\rho \leq \delta^{2} / 64 p \log (m n p)$, with high probability, one has

$$
(1-\delta)\|S\|_{F} \leq\left\|\mathscr{P}_{\mathrm{Q}} S\right\|_{F} \leq(1+\delta)\|S\|_{F} .
$$

Proof. For $\left\|\mathscr{P}_{\mathrm{Q}}\right\| \leq 1$, we have $\left\|\mathscr{P}_{\mathrm{Q}} S\right\|_{F} \leq\|S\|_{F} \leq(1+\delta)\|S\|_{F}$. On the other hand, we have

$$
\left\|\mathscr{P}_{\mathrm{Q}} S\right\|_{F}=\left\|S-\mathscr{P}_{\mathrm{Q}^{\perp}} S\right\|_{F} \geq\|S\|_{F}-\left\|\mathscr{P}_{\mathrm{Q}^{\perp}} S\right\|_{F} \geq(1-\delta)\|S\|_{F} .
$$

\subsection{Bounding the Behavior of $\left\|\mathscr{P}_{\mathrm{Q}^{\perp}}(L)\right\|_{F}$.}

Lemma 9 (see $[7,18])$. Suppose that $Y_{d}$ is distributed as a chisquared random variable with d degrees of freedom. Then, for each $t>0$

$$
\begin{gathered}
\mathbb{P}\left\{Y_{d}-d \geq t \sqrt{2 d}+t^{2}\right\} \leq e^{-t^{2} / 2}, \\
\mathbb{P}\left\{Y_{d} \leq d(1-\epsilon)\right\} \leq e^{-\epsilon^{2} d / 4} .
\end{gathered}
$$

Lemma 10. With high probability, one has

$$
\|H\|_{F} \geq \frac{1}{2} .
$$

Proof. According to the definition of $H$, we have

$$
\|H\|_{F}^{2}=\frac{\|M\|_{F}^{2}}{m n},
$$


where $(M)_{i j} \sim \mathcal{N}(0.1)$. It is necessary to bound $\|M\|_{F}^{2}$. It is easy to note that $\|M\|_{F}^{2}$ is distributed as a chi-squared random variable with $m n$ degrees of freedom. Letting $\epsilon=3 / 4$, we have

$$
\mathbb{P}\left\{Y_{d} \leq m n(1-\epsilon)\right\} \leq e^{-\epsilon^{2} m n / 4},
$$

where we have used (19). Equation (22) tells us: $\|M\|_{F}^{2} \geq m n / 4$ with high probability. Therefore, Lemma 9 is established.

Lemma 11 (see $[8,9])$. Assume that $\Omega \sim \operatorname{Ber}(\rho)$. If $1-\rho \geq$ $C \epsilon^{-2}(\mu r \log n / n)$, then with high probability

$$
\left\|\mathscr{P}_{\Omega} \mathscr{P}_{T}\right\|^{2} \leq \rho+\epsilon .
$$

According to Lemma 11, if $\epsilon$ and $\rho$ are small enough, we have the below formula with high probability

$$
\left\|\mathscr{P}_{\Omega} \mathscr{P}_{T}\right\| \leq \frac{1}{2} .
$$

Lemma 12. Under the assumption of Theorem 1, with high probability, one has

$$
\|L\|_{F}+\|S\|_{F} \leq 5\|M\|_{F} .
$$

Proof. Note that $L \in T$, and we have

$$
\begin{aligned}
\left\|\mathscr{P}_{\Omega} L\right\|_{F}^{2} & =\left\|\mathscr{P}_{\Omega} \mathscr{P}_{T} L\right\|_{F}^{2} \\
& \leq \frac{1}{4}\|L\|_{F}^{2} \\
& =\frac{1}{4}\left(\left\|\mathscr{P}_{\Omega} L\right\|_{F}^{2}+\left\|\mathscr{P}_{\Omega^{\perp}} M\right\|_{F}^{2}\right) .
\end{aligned}
$$

In the first inequality, we have used Lemma 11. In the second equality, we have used $\mathscr{P}_{\Omega^{\perp}} L=\mathscr{P}_{\Omega^{\perp}} M$, because $S \in \Omega$. It is obvious that

$$
\left\|\mathscr{P}_{\Omega} L\right\|_{F} \leq \frac{\sqrt{3}}{3}\left\|\mathscr{P}_{\Omega^{\perp}} M\right\|_{F}
$$

Therefore,

$$
\begin{aligned}
\|L\|_{F} & \leq\left\|\mathscr{P}_{\Omega} L\right\|_{F}+\left\|\mathscr{P}_{\Omega^{\perp}} L\right\|_{F} \\
& \leq \frac{\sqrt{3}}{3}\left\|\mathscr{P}_{\Omega^{\perp}} M\right\|_{F}+\left\|\mathscr{P}_{\Omega^{\perp}} M\right\|_{F} \\
& \leq \frac{5}{3}\|M\|_{F} .
\end{aligned}
$$

And thus, we can obtain

$$
\begin{aligned}
\|L\|_{F}+\|S\|_{F} & =\|L\|_{F}+\|M-L\|_{F} \\
& \leq 2\|L\|_{F}+\|M\|_{F} \\
& \leq 5\|M\|_{F} .
\end{aligned}
$$

Lemma 12 is established.
Next, we will bound the behavior of $\left\|\mathscr{P}_{\mathrm{Q}^{\perp}}(L)\right\|_{F}$, based on Lemma 9 to Lemma 12.

Theorem 13(a). Suppose that $L$ be r-rank matrix and $Q^{\perp}$ is a $p$-dimensional random subspace of $\mathbb{R}^{n \times n}$. If $r \leq\left(m n \delta^{2} / 12 p\right)$ $(5 / 3) \log (n)$, with high probability, one has

$$
\left\|\mathscr{P}_{\mathrm{Q}^{\perp}} L\right\|_{F} \leq \delta\|L\|_{F} .
$$

As pointed out by one reviewer, Lemma 15 in [10] is similar to Theorem 13(a). Lemma 15 in [10] tells us (30) is established if $r \leq n \delta^{2} / p v$; however it is obvious that $r$ is strongly based on $v$ which is defined as if there exists an orthonormal basis $\left\{G_{i}\right\}$ for $L$ satisfying $\max _{i}\left\|G_{i}\right\|^{2} \leq v / n$, which is very difficult to compute. The condition of $r$ in Theorem 13(a) is just based on $m, n, p$, and $\delta$, which is easy to determine.

Proof. According to the definition of $Q^{\perp}$, we have

$$
\left\|\mathscr{P}_{\mathrm{Q}^{\perp}} L\right\|_{F}=\sqrt{\sum_{k=1}^{p}\left|\left\langle G_{k}, L\right\rangle\right|^{2}} \leq \sqrt{p} \max _{k}\left|\left\langle G_{k}, L\right\rangle\right|,
$$

where $G_{1}, G_{2}, \ldots, G_{k}$ are independent and identically distributed to $G$ mentioned before. According to (31), it is necessary to bound $|\langle G, L\rangle|$. Suppose that $U \Sigma V^{T}$ is singular value decomposition of matrix $L$; that is, $L=U \Sigma V^{T}=$ $\sum_{l=1}^{r} \sigma_{l} u_{l} v_{l}^{T}$. Therefore, according to triangle inequality, we have

$$
\begin{aligned}
|\langle G, L\rangle| & \leq \sum_{l=1}^{r} \sigma_{l}\left|\left\langle G, u_{l} v_{l}^{T}\right\rangle\right| \\
& =\sum_{l=1}^{r} \sigma_{l}\left|u_{l}^{T} G v_{l}\right| \\
& \leq \sqrt{\sum_{l=1}^{r} \sigma_{l}^{2}} \sqrt{\sum_{l=1}^{r}\left(u_{l}^{T} G v_{l}\right)^{2}} \\
& =\frac{\|L\|_{F}}{\|H\|_{F} \sqrt{m n}} \sqrt{\sum_{l=1}^{r}\left(u_{l}^{T} M v_{l}\right)^{2}} \\
& \leq \frac{2\|L\|_{F}}{\sqrt{m n}} \sqrt{\sum_{l=1}^{r}\left(u_{l}^{T} M v_{l}\right)^{2},}
\end{aligned}
$$

where $u_{l}, v_{l}$ are the $l$ th rows of $U, V$, respectively. In the second inequality, we have used Cauchy-Schwarz inequality. In the last inequality, we have used Lemma 10. Let $y_{l}:=u_{l}^{T} M v_{l}=$ $\sum_{i j} u_{l i} m_{i j} v_{l j}$; note that

$$
\begin{aligned}
\mathbb{E}\left\{y_{l}\right\} & =\sum_{i j} u_{l i} v_{l j} \mathbb{E}\left\{m_{i j}\right\}=0, \\
\operatorname{Var}\left\{y_{l}\right\} & =\sum_{i j} \operatorname{Var}\left\{u_{l i} v_{l j} m_{i j}\right\} \\
& =\sum_{i j} u_{l i}^{2} v_{l j}^{2} \operatorname{Var}\left\{m_{i j}\right\}=1,
\end{aligned}
$$


where we have used the fact $\sum_{i} u_{l i}^{2}=1$ and $\sum_{j} v_{l j}^{2}=1$. In other word $y_{l} \sim \mathcal{N}(0,1)$. Let $z:=\sum_{l=1}^{r}\left(u_{l}^{T} M v_{l}\right)^{2}=\sum_{l=1}^{r} y_{l}^{2}$. It is obvious that $z$ is distributed as a chi-squared random variable with $r$ degrees of freedom.

According to Lemma 9, letting $t=2 \sqrt{\log n}$, we can obtain

$$
\mathbb{P}\left(Y_{d} \geq d+2 \sqrt{\log n} \sqrt{2 d}+4 \log n\right) \leq n^{-2} .
$$

It implies that with high probability

$$
Y_{d} \leq d+2 \sqrt{\log n} \sqrt{2 d}+4 \log n
$$

That is, with high probability

$$
z \leq r+2 \sqrt{\log n} \sqrt{2 r}+4 \log n .
$$

Combining with (31), (32), and (36), with high probability, we can obtain

$$
\begin{aligned}
\left\|\mathscr{P}_{\mathrm{Q}^{\perp}} L\right\|_{F} & \leq \frac{2 \sqrt{p}\|L\|_{F}}{\sqrt{m n}} \sqrt{r+2 \sqrt{\log n} \sqrt{2 r}+4 \log n} \\
& \leq \frac{2 \sqrt{p}\|L\|_{F}}{\sqrt{m n}} \sqrt{3 r+5 \log n},
\end{aligned}
$$

where we have used the fact: if $a, b \geq 0,2 \sqrt{a b} \leq a+b$. Let

$$
\frac{2 \sqrt{p}}{\sqrt{m n}} \sqrt{3 r+5 \log n} \leq \delta .
$$

That is,

$$
r \leq \frac{m n \delta^{2}}{12 p}-\frac{5}{3} \log (n) .
$$

Theorem 13(a) is established.

Theorem 13(b). Suppose that $L$ is $\mu$-incoherent matrix and $Q^{\perp}$ is a p-dimensional random subspace of $\mathbb{R}^{n \times n}$. If $r \leq$ $\left(m n \delta^{2} / 12 p\right)-(5 / 3) \log (n)$, with high probability, one has

$$
(1-\delta)\|L\|_{F} \leq\left\|\mathscr{P}_{\mathrm{Q}} L\right\|_{F} \leq(1+\delta)\|L\|_{F} .
$$

Proof. The main idea of the proof follows the arguments of Theorem 8(b).

Proof of Theorem 4. According to Theorems 8 and 13 and the assumption of Lemma 3, we have

$$
\begin{gathered}
\left\|\mathscr{P}_{\mathrm{Q}^{\perp}} L\right\|_{F} \leq \delta\|L\|_{F}, \\
\left\|\mathscr{P}_{\mathrm{Q}^{\perp}} S\right\|_{F} \leq \delta\|S\|_{F} .
\end{gathered}
$$

Therefore,

$$
\begin{aligned}
\left\|\mathscr{P}_{\mathrm{Q}^{\perp}}(L+S)\right\|_{F} & \leq\left\|\mathscr{P}_{\mathrm{Q}^{\perp}} L\right\|_{F}+\left\|\mathscr{P}_{\mathrm{Q}^{\perp}} S\right\|_{F} \\
& \leq \delta\left(\|L\|_{F}+\|S\|_{F}\right) \\
& \leq 5 \delta\|M\|_{F} .
\end{aligned}
$$

Then,

$$
\begin{aligned}
\left\|\mathscr{P}_{\mathrm{Q}} M\right\|_{F} & =\left\|M-\mathscr{P}_{\mathrm{Q}^{\perp}} M\right\|_{F} \\
& \geq\|M\|_{F}-\left\|\mathscr{P}_{\mathrm{Q}^{\perp}} M\right\|_{F} \\
& \geq(1-5 \delta)\|M\|_{F} .
\end{aligned}
$$

Theorem 4 is established.

\section{Conclusion}

In this paper, we address the problem of principal component pursuit with reduced linear measurements (PCP_RLM). In order to obtain an easy handed band of $\tau$, we prove that operator $\mathscr{P}_{Q}$ obeys RIP with high probability if the low-rank matrix satisfies incoherent conditions and the sparse matrix is enough sparse. And then, based on the RIP of operator $\mathscr{P}_{\mathrm{Q}}$, we provide the bound of parameters depending only on observed quantities. In the future, we will try to analyze the robustness of optimum solution of the strongly convex programming (1) with RIP property.

\section{Acknowledgments}

The authors would like to thank the anonymous reviewers for their comments that helped to improve the quality of the paper. This research was supported by the National Natural Science Foundation of China (NSFC) under Grant 61172140, and "985" Key Projects for the excellent teaching team support (postgraduate) under Grant A1098522-02. Haiwen $\mathrm{Xu}$ is supported by Joint Fund of the National Natural Science Foundation of China and the Civil Aviation Administration of China (Grant no. U1233105).

\section{References}

[1] J. Ellenberg, "Fill in the blanks: using math to turn lo-res datasets into hi-ressamples," http://www.wired.com/magazine/ 2010/02/ff_algorithm/.

[2] A. Chambolle and P.-L. Lions, "Image recovery via total variation minimization and related problems," Numerische Mathematik, vol. 76, no. 2, pp. 167-188, 1997.

[3] J. F. Claerbout and F. Muir, "Robust modeling of erratic data," Geophysics, vol. 38, pp. 826-844, 1973.

[4] B. Zeng and J. Fu, "Directional discrete cosine transformsa new framework for image coding," IEEE Transactions on Circuits and Systems for Video Technology, vol. 18, no. 3, pp. 305313, 2008.

[5] M. Elad and M. Aharon, "Image denoising via sparse and redundant representations over learned dictionaries," IEEE Transactions on Image Processing, vol. 15, no. 12, pp. 3736-3745, 2006.

[6] H. Ji, C. Q. Liu, Z. W. Shen, and Y. Xu, "Robust video denoising using Low rank matrix completion," in Proceedings of the IEEE Computer Society Conference on Computer Vision and Pattern Recognition (CVPR '10), pp. 1791-1798, June 2010.

[7] E. J. Candès and B. Recht, "Exact matrix completion via convex optimization," Foundations of Computational Mathematics, vol. 9, no. 6, pp. 717-772, 2009. 
[8] E. J. Candès, X. Li, Y. Ma, and J. Wright, "Robust principal component analysis?" Journal of the ACM, vol. 58, no. 3, article 11, 2011.

[9] Z. Zhou, X. Li, J. Wright, E. Candès, and Y. Ma, "Stable principal component pursuit," in Proceedings of the IEEE International Symposium on Information Theory (ISIT '10), pp. 1518-1522, June 2010.

[10] A. Ganesh, K. Min, J. Wright, and Y. Ma, "Principal component pursuit with reduced linear measurements," IEEE International Symposium on Information Theory Proceedings, pp. 1281-1285, 2012.

[11] J. Wright, A. Ganesh, K. Min, and Y. Ma, "Compressive principal component pursuit," IEEE International Symposium on Information Theory Proceedings, pp. 1276-1280, 2012.

[12] H. Zhang, J.-F. Cai, L. Cheng, and J. Zhu, "Strongly convex programming for exact matrix completion and robust principal component analysis," Inverse Problems and Imaging, vol. 6, no. 2, pp. 357-372, 2012.

[13] B. Recht, M. Fazel, and P. A. Parrilo, "Guaranteed minimumrank solutions of linear matrix equations via nuclear norm minimization," SIAM Review, vol. 52, no. 3, pp. 471-501, 2010.

[14] Q. S. You, Q. Wan, and Y. P. Liu, "A short note on strongly convex programming for exact matrix completion and robust principal component analysis," Inverse Problems and Imaging, vol. 7, no. 1, pp. 305-306, 2013.

[15] Q. S. You and Q. Wan, "Strongly convex programming for principal component pursuit," http://arxiv.org/abs/1209.4405.

[16] R. Meka, P. Jain Inderjit, and S. Dhillon, "Guaranteed rank minimization via singular value projection," in Neural Information Processing Systems, 2010.

[17] L. Mackey, M. I. Jordan, R. Y. Chen, B. Farrell, and J. A. Tropp, "Matrix concentration inequalities via the method of exchangeable Pairs," http://arxiv.org/abs/1201.6002.

[18] B. Laurent and P. Massart, "Adaptive estimation of a quadratic functional by model selection," The Annals of Statistics, vol. 28, no. 5, pp. 1302-1338, 2000. 


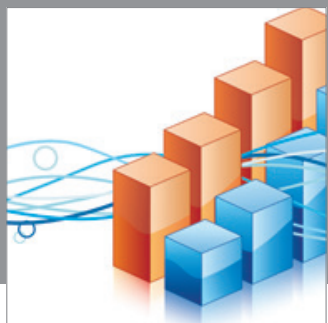

Advances in

Operations Research

mansans

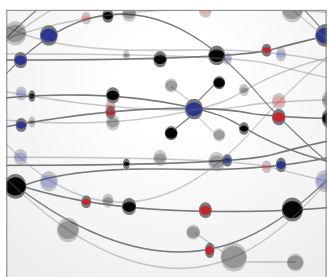

The Scientific World Journal
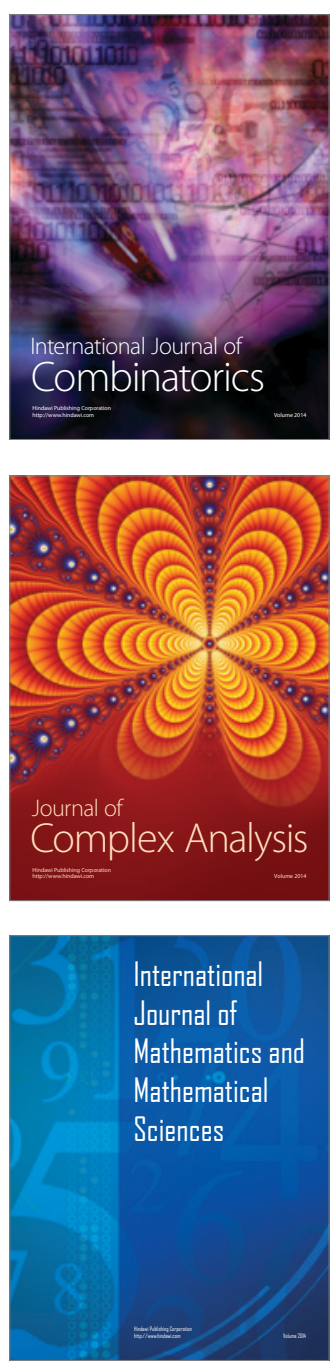
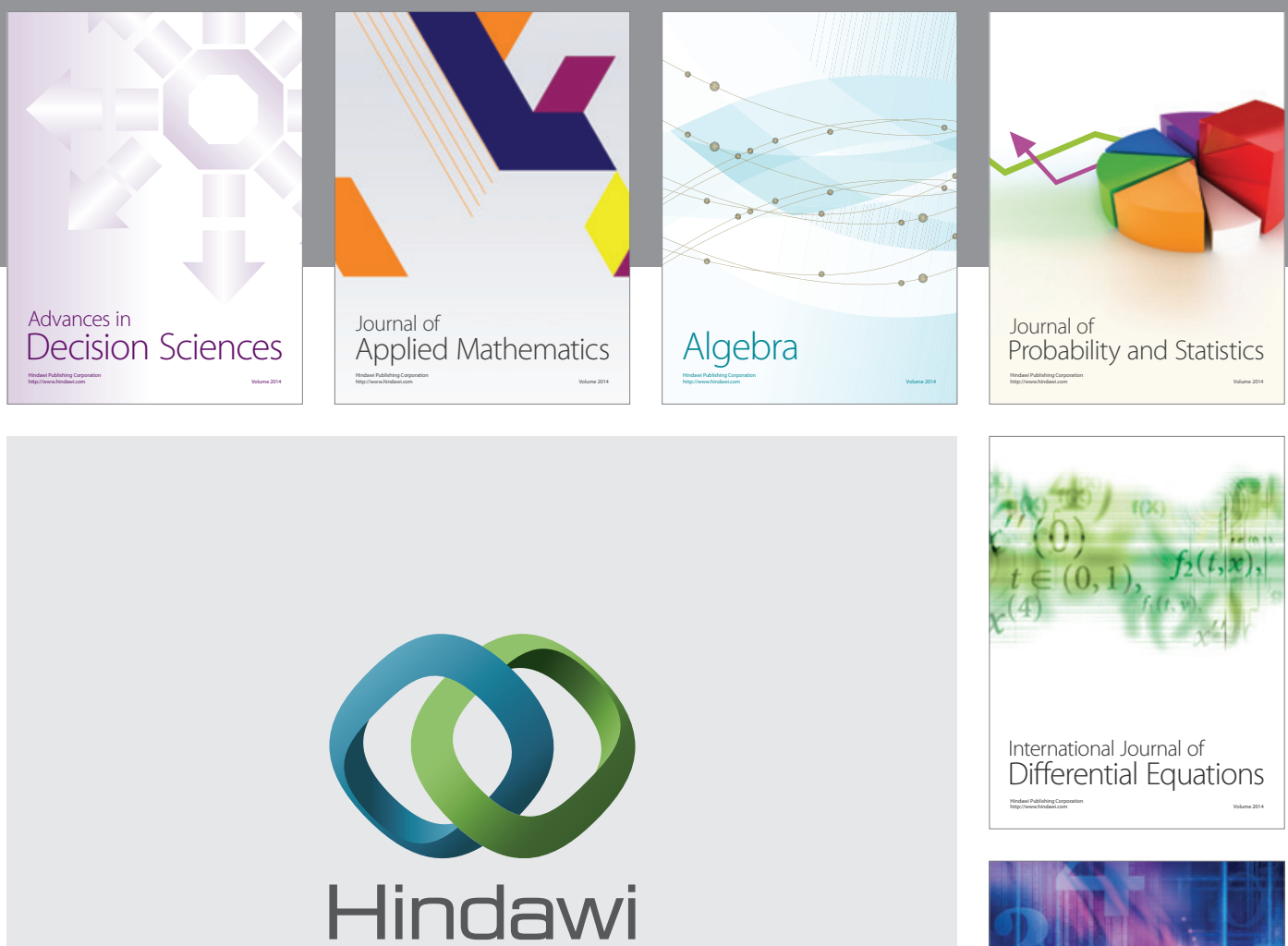

Submit your manuscripts at http://www.hindawi.com
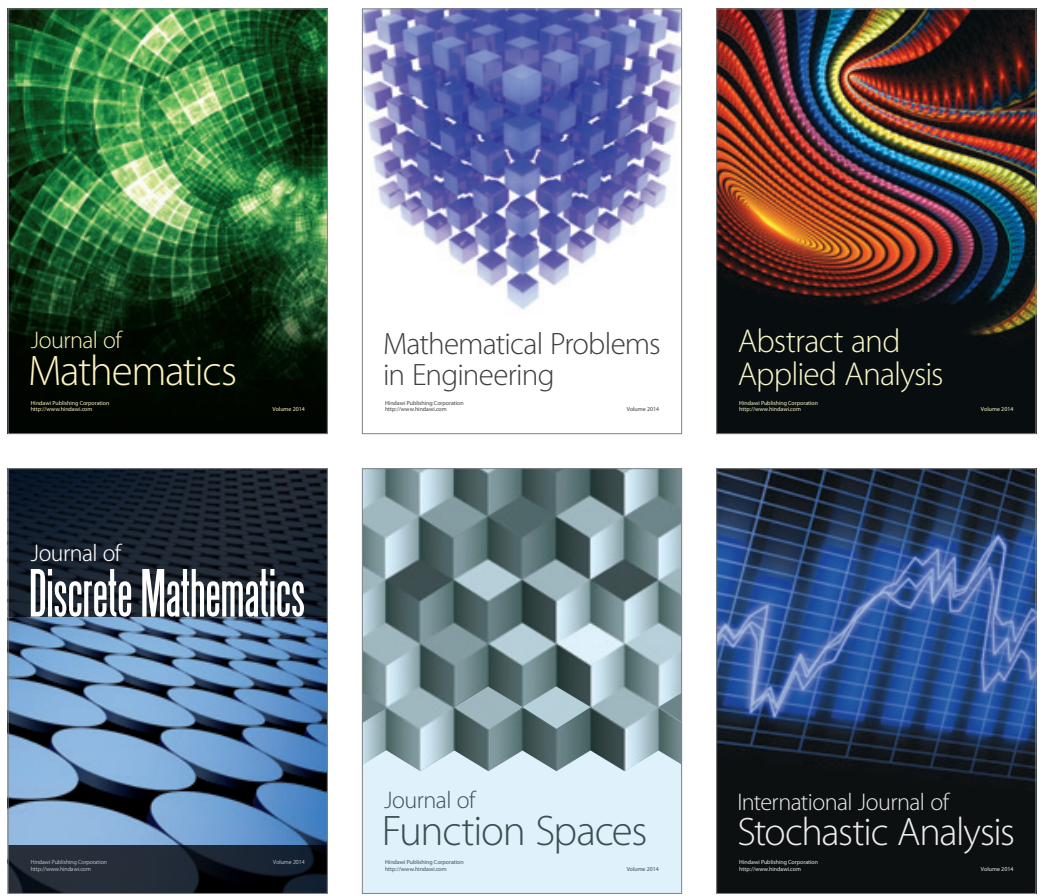

Journal of

Function Spaces

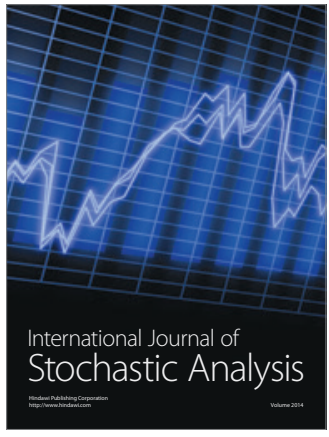

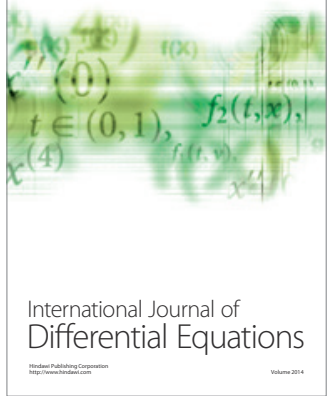
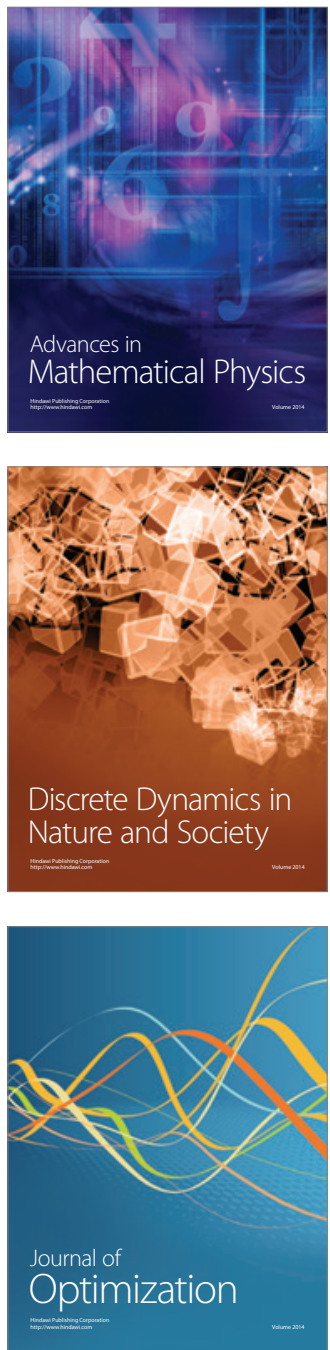